FINANCIAL PROGNOSIS: REGRESSION AND CORRELATION METHOD. CASE: MANUFACTURING COMPANY

\title{
PRONÓSTICOS FINANCIEROS: MÉTODO DE REGRESIÓN Y CORRELACIÓN. CASO: EMPRESA MANUFACTURERA
}

\section{Ph.D Saury José Thomas Manzano*, MSc (c) Sandra Milena Castro Escobar*, MSc (c) Richard Antonio Moya Argüello*}

*Universidad de Pamplona, Ingenierías y Arquitectura, Ingeniería Industrial, Grupo de investigación (Investigación, Gestión y Administración de Producción y Operaciones)

\section{INGAPO}

Ciudadela Universitaria. Pamplona, Norte de Santander, Colombia.

Tel.: 57-7-5685303, Fax: 57-7-5685303, Ext. 144

E-mail: \{saurythomas, sandra.castro, richard_moya\}@ unipamplona.edu.co

Resumen: A partir de datos con tendencia lineal, se presentan en este artículo los cálculos de pronóstico a través del método de regresión y correlación verificada a través de la correlación, en empresas manufactureras, método que adquiere importancia cuando los datos exceden los 11 años, esta metodología permite la interpretación de resultados mediante una estimación rápida de las ventas, cuentas por cobrar e inventarios en los próximos 4 años, 2016, 2017, 2018 y 2019, logrando establecer un parámetro de referencia para que la alta dirección tome decisiones acertadas en materia de inversión y financiamiento.

Palabras clave: Tendencia, regresión, correlación, estimación, inventarios, inversión y financiamiento.

\begin{abstract}
From data with linear trend, are presented in this article estimates forecast by the method of regression and correlation verified through correlation, in manufacturing, method becomes important when the data exceeds 11 years, this methodology allows the interpretation of results through a quick estimate of sales, accounts receivable and inventories over the next four years, 2016, 2017, 2018 and 2019, managing to establish a benchmark for top management make sound decisions on investment and financing.
\end{abstract}

Keywords: Trend forecasts, regression, correlation, inventories, financing. 


\section{INTRODUCCIÓN}

Las empresas deben poseer activos para hacer ventas y si las ventas han de tender positivamente los activos por consiguiente aumentan. La búsqueda para ampliar las ventas requiere de una inversión inmediata en nuevos activos y a medida que se agote la capacidad total, deben adquirirse nuevos activos circulantes, este último llamado también Activo Corriente dado que está formado por los bienes y derechos que son líquidos o pueden convertirse en dinero en un corto plazo. Estos son: El efectivo, Cuentas por cobrar e Inventarios.

Los activos deben ser financiados por consiguiente implica adquirir compromisos y obligaciones. Es preocupación de las empresas determinar la necesidad de fondos para sostener las operaciones comerciales.

El método de regresión es más general. Calcula las relaciones promedio a lo largo de un periodo de tiempo; por lo tanto, no depende tanto de los datos actuales de un punto particular en el tiempo y es generalmente más exacto si se proyecta una tasa de crecimiento considerable o si el periodo del pronóstico abarca varios años. La esencia el método de regresión se ilustra con el diagrama de dispersión.

El método de regresión es exacto por tender a un alto nivel de correlación cuando tiene un número mayor o igual a 11 datos, para ese tipo de cálculo se pueden utilizar programas como el SPSS o incluso Excel para apoyar el modelo matemático de cálculo manual como el presentado en este artículo. Un pronóstico puede definirse como el resultado de la aplicación de un método de predicción (como la extrapolación lineal) en que partiendo de determinadas series de datos (cuya selección deberá realizarse también según reglas metódicas), se formula una "proyección" en el futuro con el objetivo de evaluar la ocurrencia probable de cualquier acontecimiento o el desarrollo de una tendencia ${ }^{1}$.

Para pronosticar la demanda de un producto o servicio, existen diversas maneras de hacerlo. En esta ocasión se presenta un proceso sencillo para visualizar el posible comportamiento de la demanda empleando la Regresión Lineal, la cual nos permitirá observar la Línea de Tendencia. Como caso práctico, tenemos una empresa manufacturera que se encuentra realizando un estudio de mercado para ofrecer sus productos.

\footnotetext{
${ }^{1}$ http://sunwc.cepade.es/ jrivera/org_temas/metodos/prospectiva/pronostic_method.htm
}

Para ello, se ha estudiado y recolectado información de ventas, cuentas por cobrar e inventario en un periodo de 11 años comprendido entre los años 2005 -2015. Ahora, con estos datos queremos conocer cómo es el comportamiento o tendencias para los años futuros 20016, 2017, 2018, 2019 y poder anticiparnos a través de una planeación de los diferentes recursos que necesitaremos para responder efectivamente a la demanda esperada. Además de ello apoyar la toma de decisiones en distintas áreas de la dirección de empresas: el pronóstico de ventas ayudará a diseñar el plan de producción, el pronóstico de evolución de precios de materias primas, suministros, mayor flexibilidad en la elaboración de los planes, predecir el desarrollo futuro (del sistema, de su entorno etc.) Medidas de apoyo, contramedidas u otras acciones que influyan, en mayor o menor grado, sobre la tendencia del objeto, garantizando el crecimiento, desarrollo y sostenibilidad de la empresa a través del tiempo.

\section{METODOLOGÍA}

Para realizar los pronósticos correspondientes a las Ventas, Inventarios y Cuentas por Cobrar es necesario tener la información histórica de cada una de ellas. Datos presentados en la tabla 1:

\section{Tabla 1: Histórico de ventas, Inventarios y cuentas} por cobrar

\begin{tabular}{|c|c|c|c|}
\hline \multicolumn{5}{|c|}{$\begin{array}{c}\text { DATOS HIS TORICOS EMPRESA MANUFACTURERA, VENTAS, CUENTAS } \\
\text { POR COBRAR E INVENTARIOS. }\end{array}$} \\
\hline \multicolumn{4}{|c|}{ EN MILLONES DE DOLARES } \\
\hline AÑOS & VENTAS & INVENTARIOS & CUENTAS POR COBRAR \\
\hline 2.005 & 175 & 44 & 34 \\
\hline 2.006 & 200 & 48 & 38 \\
\hline 2.007 & 215 & 53 & 42 \\
\hline 2.008 & 185 & 57 & 42 \\
\hline 2.009 & 235 & 60 & 44 \\
\hline 2.010 & 265 & 66 & 42 \\
\hline 2.011 & 300 & 73 & 52 \\
\hline 2.012 & 280 & 70 & 43 \\
\hline 2.013 & 350 & 78 & 62 \\
\hline 2.014 & 420 & 90 & 75 \\
\hline 2.015 & 500 & 100 & 85 \\
\hline
\end{tabular}

Fuente: autores

Dada esta información se pronosticaron por método de regresión las Ventas, Cuentas por Cobrar e Inventarios, para los años 2016, 2017, 2018,2019 , con el fin de soportar cada uno de los resultados no solo se realizó el cálculo de correlación sino además se comprobó utilizando las herramientas IBM SPSS y Microsoft Excel.

A través del cálculo realizado para el pronóstico de las ventas 2016, 2017, 2018 y 2019 se dio la base para poder analizar y calcular los pronóstico de las Cuentas por Cobrar e Inventarios. 


\section{RESULTADOS Y DISCUSIÓN}

Los beneficios de un proceso que está preparando a las cambios bruscos de la demanda e identifica el comportamiento de sus clientes, es capaz de amortiguar las variaciones bruscas del mercado, analizando sus tendencias, financieramente está preparado para soportar los gastos directos e indirectos que sugiere preparar un lote de producción y de la mano para evaluar ese análisis efectivo del valor ganado se deben responder tres preguntas ¿Cuál fue el trabajo planificado?, ¿Cuánto trabajo se ha completado? y ¿cuál es el costo en el que se ha incurrido?. (Serrano, 2014)

Es de destacar que el fracaso financiero puede deberse a la ausencia de un estudio organizacional meticuloso donde no se le ha dado la importancia en términos de solvencia y de equilibrio en todas las áreas del negocio en cada empresa proyectando sus necesidades económicas. (Monelos, P., et al 2016)

Entonces se establece como parte de una Prospectiva Financiera, dado que se anticipa el riesgo operativo que implica producir un mínimo de unidades, allí es donde radica la importancia de la relación pronóstico financiero y la prospectiva. (Gonzales 2017, et al. )

Los datos de Ventas, Cuentas por Cobrar e Inventarios se presentan de forma agrupada con el fin de calcular en un comienzo el modelo de Regresión que se utilizará para los respectivos pronósticos. Para apoyar la veracidad de los resultados se procedió a calcular la correlación verificando dicho guarismo a través del uso de la herramienta hoja de cálculo Microsoft Excel, ver Tabla 2.

\subsection{PRONÓSTICO AÑOS Vs VENTAS EN MILLONES DE DOLARES}

Se realizaron los cálculos de manera agrupada para definir los coeficientes que harán parte del modelo para el pronóstico utilizando las ecuaciones 1, 2 y 3.

Tabla 2: Histórico de ventas

\begin{tabular}{|c|c|c|c|c|}
\hline AÑOS & VENTAS & \multirow{2}{*}{$\mathbf{X Y}$} & \multirow{2}{*}{$\mathbf{\wedge} \mathbf{2}$} & \multirow{2}{*}{$\mathbf{Y}^{\wedge} \mathbf{2}$} \\
\hline $\mathbf{X}$ & $\mathbf{Y}$ & 175 & 1 & 30.625 \\
\hline 1 & 175 & 4 & 4 & 40.000 \\
\hline 2 & 200 & 400 & 9 & 46.225 \\
\hline 3 & 215 & 645 & 9 & 34.225 \\
\hline 4 & 185 & 740 & 16 & 55.225 \\
\hline 5 & 235 & 1,175 & 25 & 70 \\
\hline 6 & 265 & 1,590 & 36 & 70.225 \\
\hline 7 & 300 & 2,100 & 49 & 90.000 \\
\hline 8 & 280 & 2,240 & 64 & 78.400 \\
\hline 9 & 350 & 3,150 & 81 & 122.500 \\
\hline 10 & 420 & 4,200 & 100 & 176.400 \\
\hline 11 & 500 & 5,500 & 121 & 250.000 \\
\hline $\mathbf{6 6}$ & $\mathbf{3 . 1 2 5}$ & $\mathbf{2 1 . 9 1 5}$ & $\mathbf{5 0 6}$ & $\mathbf{9 9 3 . 8 2 5}$ \\
\hline
\end{tabular}

Fuente: autores

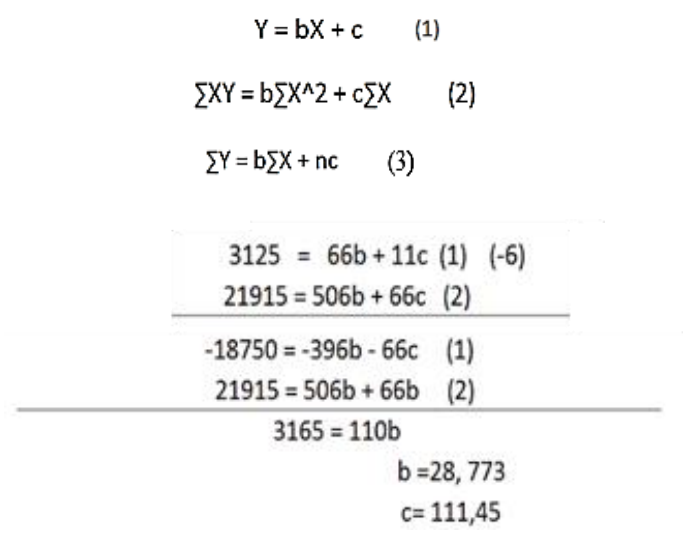

Con la ecuación 4 y 5 se calcularon las varianzas con el fin de hallar la covarianza del conjunto de datos analizados.

$$
\begin{aligned}
& S x^{\wedge} 2=\Sigma X_{i}^{\wedge} 2-n \bar{X}^{\wedge} 2 \\
& =\underline{506-(11)\left(6^{\wedge} 2\right)}=10 \\
& 11 \\
& S y^{\wedge} 2=\sum Y_{i}^{\wedge} 2-n \bar{Y}^{\wedge} 2 \\
& n \\
& =\underline{993825-(11)\left(284,0909^{\wedge} 2\right)}=9640.0878
\end{aligned}
$$$$
11
$$

Se calcula la covarianza, Ecuación 6

$$
\begin{aligned}
& \text { Covarianza }=\underset{n x y}{\operatorname{SXY}}-\bar{X} \bar{Y} \\
& =\frac{21915}{11}-(6)(284,0909)=287,7273
\end{aligned}
$$

Se calculó el coeficiente de correlación mediante la ecuación 7

$$
\begin{aligned}
R^{\wedge} 2= & \frac{(\text { Covarianza })^{\wedge} 2}{\left(S_{x} \wedge 2\right)\left(S^{\wedge} 2\right)} \\
& =\frac{(287,7273)^{\wedge} 2}{10(9640,0878)}=0,8588
\end{aligned}
$$

Utilizando el modelo (Ecuación 8) se calculó el pronóstico de los siguientes 4 años:

$$
\hat{Y}=28,773 X+111.45
$$

$$
\begin{aligned}
& \hat{Y}_{12}=457 \\
& \hat{Y}_{13}=486 \\
& \hat{Y}_{14}=514 \\
& \hat{Y}_{15}=543
\end{aligned}
$$

Haciendo uso del SPSS software se obtiene: 
Tabla 3: Resumen del modelo

\begin{tabular}{|c|c|c|c|c|}
\hline Modelo & $\mathbf{R}$ & $\mathbf{R}$ cuadrado & $\begin{array}{c}\text { R cuadrado } \\
\text { corregida }\end{array}$ & $\begin{array}{c}\text { Error típ. de } \\
\text { la estimación }\end{array}$ \\
\hline 1 &, $927^{\mathrm{a}}$ &, 859 &, 843 & 40,79110 \\
\hline
\end{tabular}

a. Variables predictoras: (Constante), AÑOS

Fuente: autores

Nuevamente a través del software comprueban los coeficientes estandarizados que fueron calculados anteriormente:

Tabla 4: Coeficientes

\begin{tabular}{|c|c|c|c|c|c|}
\hline \multirow{2}{*}{ Modelo } & \multicolumn{2}{|c|}{ Coeficientes no estandarizados } & \multirow{2}{*}{$\begin{array}{c}\begin{array}{c}\text { Coefícientes } \\
\text { tipincados }\end{array} \\
\text { Beta } \\
\end{array}$} & \multirow{2}{*}{$t$} & \multirow{2}{*}{ Sig. } \\
\hline & B & Errortip. & & & \\
\hline $1 \quad \begin{array}{c}\text { (Constante) } \\
\text { AÑos }\end{array}$ & $\begin{array}{l}111,455 \\
28,773\end{array}$ & $\begin{array}{c}26,378 \\
3889\end{array}$ & 927 & $\begin{array}{l}4,225 \\
7,398\end{array}$ & .002 \\
\hline
\end{tabular}

a. Variable dependiente: VENTAS

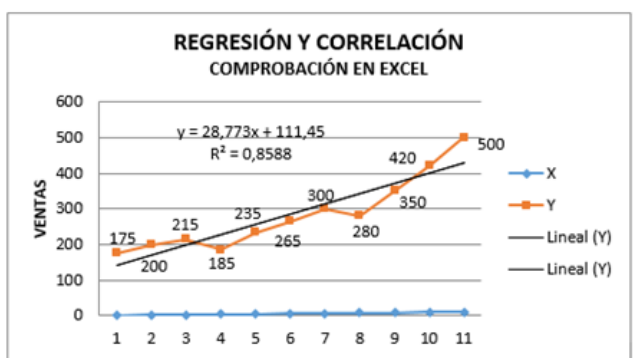

Figura 1. Comprobación software Excel Ventas Fuente: autores

Se obtuvo una correlación de 0,85 definiendo al conjunto de datos tomado para la investigación con una buena relación de variables

\subsection{PRONÓSTICO VENTAS VS INVENTARIOS EN MILLONES DE DOLARES}

Siendo $\mathbf{X}$ ventas como variable independiente y $\mathbf{Y}$ inventarios, como variable dependiente, además se tiene en cuenta que para realizar el pronóstico los valores de $\mathrm{x}$ corresponden a las ventas de los años 2016, 2017, 2018 y 2019. Tabla 5.

\section{Tabla 5: Cálculo de las variables para Ventas Vs}

Inventarios

\begin{tabular}{|c|c|c|c|c|c|}
\hline AÑOS & $\begin{array}{c}\text { VENTAS } \\
\mathrm{X}\end{array}$ & \begin{tabular}{|c|} 
INVENTARIO \\
$\mathrm{Y}$
\end{tabular} & $X Y$ & $x^{\wedge} 2$ & $Y^{\wedge} 2$ \\
\hline 2.005 & 175 & 44 & 7.700 & 30.625 & 1.936 \\
\hline 2.006 & 200 & 48 & 9.600 & 40.000 & 2.304 \\
\hline 2.007 & 215 & 53 & 11.395 & 46.225 & 2.809 \\
\hline 2.008 & 185 & 57 & 10.545 & 34.225 & 3.249 \\
\hline 2.009 & 235 & 60 & 14.100 & 55.225 & 3.600 \\
\hline 2.010 & 265 & 66 & 17.490 & 70.225 & 4.356 \\
\hline 2.011 & 300 & 73 & 21.900 & 90.000 & 5.329 \\
\hline 2.012 & 280 & 70 & 19.600 & 78.400 & 4.900 \\
\hline 2.013 & 350 & 78 & 27.300 & 122.500 & 6.084 \\
\hline 2.014 & 420 & 90 & 37.800 & 176.400 & 8.100 \\
\hline 2.015 & 500 & 100 & 50.000 & 250.000 & 10.000 \\
\hline 22.088 & 3.125 & 739 & 227.430 & 993.825 & 52.667 \\
\hline
\end{tabular}

Fuente: autores
Realizando los cálculos con las fórmulas 1 al 7 se obtiene:

$$
\begin{aligned}
739 & =3.125 b+11 c \\
227.430 & =993.825 b+3.125 c
\end{aligned}
$$

$$
\begin{array}{ll}
-209.943,1818=-887.784,0909 b-3.125 c(1) & \\
227.430=993.825 b+3.125 c \quad \text { (2) } & \\
\hline 17.486,8182=106.040,9091 b & \\
& b=0,1649 \\
& c=20,333
\end{array}
$$

Se precedió a calcular la covarianza

$$
\begin{aligned}
& S x^{\wedge} 2=\Sigma X^{\wedge} 2-n \bar{X}^{\wedge} 2 \\
& n \\
& =\underline{993.825-(11)\left(284,0909^{\wedge} 2\right)}=9.640,0826 \\
& 11 \\
& S y^{\wedge} 2=\sum Y_{i}^{\wedge} 2-n \bar{Y} \bar{Y}^{\wedge} \\
& =\underline{52.667-(11)\left(67,1818^{\wedge} 2\right)}=274,5124 \\
& 11 \\
& \text { Covarianza }=M x y=\sum X Y-\bar{X} \bar{Y} \\
& \mathrm{n} \\
& =\underline{227.430}-(284,0909)(67,1818)=1.589,7108 \\
& 11 \\
& R^{\wedge} 2=\text { (Covarianza)^2 } \\
& \left(S x^{\wedge} 2\right)\left(S \gamma^{\wedge} 2\right) \\
& R^{\wedge} 2=\frac{(\text { Covarianza })^{\wedge} 2}{\left(S x^{\wedge} 2\right)\left(S y^{\wedge} 2\right)}=0,955
\end{aligned}
$$

Se establece el modelo:

$$
\hat{Y}=0,1649 X+20,333
$$

Con el modelo de regresión lineal se realiza el pronóstico de los siguientes 4 años:

$$
\begin{aligned}
& \hat{Y}_{12}=96 \\
& \hat{Y}_{13}=100 \\
& \hat{Y}_{14}=105 \\
& \hat{Y}_{15}=110
\end{aligned}
$$


Con el SPSS software y Excel se tiene:

\section{Tabla 6: Resumen del modelo}

\begin{tabular}{|c|c|c|c|c|}
\hline Modelo & $R$ & R cuadrado & $\begin{array}{c}\text { R cuadrado } \\
\text { corregida }\end{array}$ & $\begin{array}{c}\text { Error típ. de la } \\
\text { estimación }\end{array}$ \\
\hline 1 &, $977^{\mathrm{a}}$ &, 955 &, 944 & 40,79110 \\
\hline \multicolumn{4}{c}{$\begin{array}{r}\text { a. Variables predictoras: (Constante), AÑOS } \\
\text { b. Variable dependiente: VENTAS }\end{array}$}
\end{tabular}

Fuente: autores

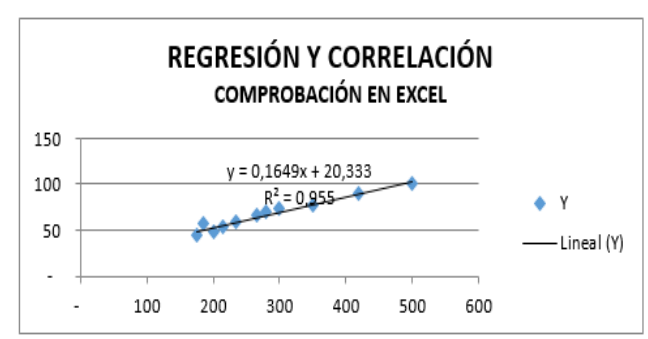

Figura 2. Comprobación software Excel Inventarios. Fuente: autores

Se obtiene una correlación excelente entre los datos

\subsection{PRONÓSTICO VENTAS Vs CUENTAS POR COBRAR EN MILLONES DE DOLARES}

Siendo $\mathrm{X}$ ventas como variable independiente y $\mathrm{Y}$ cuentas por cobrar como variable dependiente y teniendo en cuenta que para realizar el pronóstico los valores de $\mathrm{x}$ corresponden a las ventas de los años 2016, 2017, 2018 y 2019.

Tabla 7: Cálculo de las variables para Ventas Vs Cuentas por cobrar

\begin{tabular}{|c|c|c|c|c|c|}
\hline \multirow{2}{*}{ ANOS } & VENTAS & $\begin{array}{c}\text { CUENTAS } \\
\text { POR } \\
\text { COBRAR } \\
\text { (CXC) }\end{array}$ & \multirow{2}{*}{$\mathbf{X Y}$} & \multirow{2}{*}{$\mathbf{X}^{\wedge} \mathbf{2}$} & \multirow{2}{*}{$\mathbf{Y} \mathbf{2}$} \\
\cline { 2 - 5 } & $\mathbf{X}$ & $\mathbf{Y}$ & & & \\
\hline 2.003 & 175 & 34 & 5.950 & 30.625 & 1.156 \\
\hline 2.004 & 200 & 38 & 7.600 & 40.000 & 1.444 \\
\hline 2.005 & 215 & 42 & 9.030 & 46.225 & 1.764 \\
\hline 2.006 & 185 & 42 & 7.770 & 34.225 & 1.764 \\
\hline 2.007 & 235 & 44 & 10.340 & 55.225 & 1.936 \\
\hline 2.008 & 265 & 42 & 11.130 & 70.225 & 1.764 \\
\hline 2.009 & 300 & 52 & 15.600 & 90.000 & 2.704 \\
\hline 2.010 & 280 & 43 & 12.040 & 78.400 & 1.849 \\
\hline 2.011 & 350 & 62 & 21.700 & 122.500 & 3.844 \\
\hline 2.012 & 420 & 75 & 31.500 & 176.400 & 5.625 \\
\hline 2.013 & 500 & 85 & 42.500 & 250.000 & 7.225 \\
\hline $\mathbf{2 2 . 0 8 8}$ & 3.125 & 559 & $\mathbf{1 7 5 . 1 6 0}$ & 993.825 & 31.075 \\
\hline
\end{tabular}

Fuente: autores

Realizando los cálculos con las fórmulas 1 al 7 se obtiene:

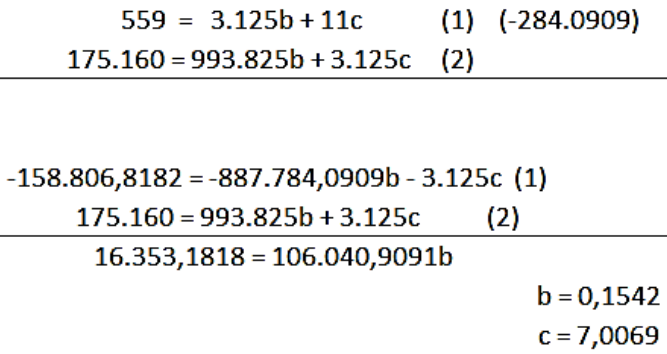

Se calcula las varianza y covariancias respectivas utilizando las ecuaciones 1 al 7

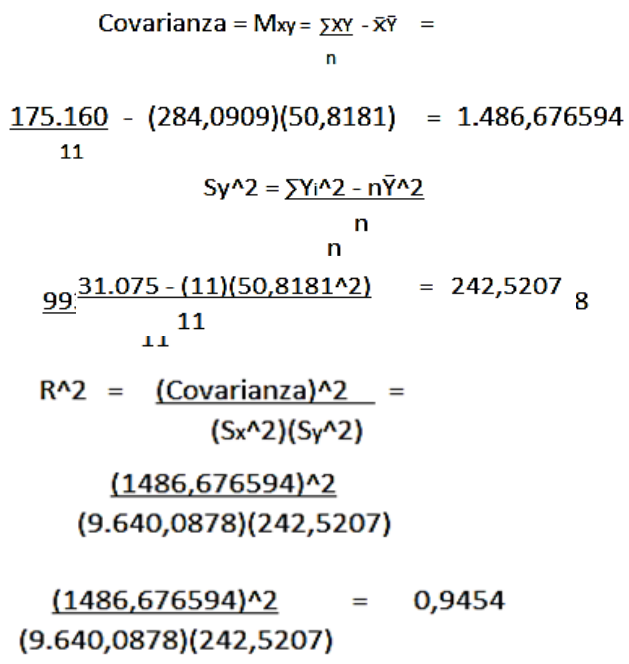

Se define el modelo

$$
\hat{Y}=0,1542 X+7,0069
$$

Encontrado el modelo de regresión lineal se realiza el pronóstico de los siguientes 4 años:

$$
\begin{aligned}
& \hat{Y}_{2014}=78 \\
& \hat{Y}_{2015}=82 \\
& \hat{Y}_{2016}=86 \\
& \hat{Y}_{2017}=91
\end{aligned}
$$

Con el SPSS software y Excel se comprueba el modelo:

Tabla 8: Resumen del modelo

\begin{tabular}{|c|c|c|c|c|}
\hline Modelo & $\mathrm{R}$ & $\mathrm{R}$ cuadrado & $\begin{array}{c}\text { R cuadrado } \\
\text { corregida }\end{array}$ & $\begin{array}{c}\text { Error típ. de la } \\
\text { estimación }\end{array}$ \\
\hline 1 &, $972^{\mathrm{a}}$ &, 945 &, 939 & 4,02379 \\
\hline
\end{tabular}

a. Variables predictoras: (Constante), VENTAS

Fuente: autores 


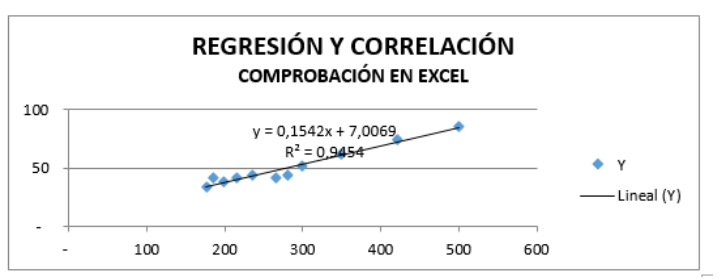

Figura 3. Comprobación software Excel Inventarios. Fuente: autores

El nivel de correlación entre las variables es excelente. A medida que el coeficiente de correlación al cuadrado disminuye, se aleja de uno, se dice también, que la recta representa cada vez menos a ese conjunto de observaciones. Cuando se presenta los siguientes intervalos: $\mathrm{R} 2=1$ es una correlación perfecta $0,9 \leq \mathrm{R} 2<1$, se dice que existe una correlación excelente, en el intervalo 0,8 $\leq \mathrm{R} 2<0,9$, se dice que existe una correlación aceptable. Entre el intervalo 0,6 $\leq \mathrm{R} 2<0,8$ se dice que hay una correlación regular. Entre el intervalo $0,3 \leq \mathrm{R} 2<0,6$ se dice que existe una correlación mínima. Y un R2<0,30 nos estará indicando que no hay correlación entre las variables. ${ }^{2}$

Es importante tener en cuenta que las empresas de no incorporar nuevos sistemas que le permitan el manejo de sus datos históricos como ventas, inventarios y su contabilidad orientando a tener una planeación con proyecciones en definitiva no lograrán ser mejor que su competencia. (Peñaranda, 2014)

Se entiende que las organizaciones entran en un estado de tensión llamado Estrés Financiero (Mejía, G 2016) cuando no se ha planeado la forma en que se manejarán los cambios económicos que afectan el balance y los malestares llegan en la medida en que se dejen pasar las oportunidades de negocio, así el efecto es desbastador en términos de rentabilidad, de allí surgen varios cuestionamientos relacionados con la productividad de los empleados. ¿Se motivan a alcanzar sus objetivos? ¿Son conscientes de la importancia de su trabajo, cuando tienen presente los resultados financieros de la empresa?, ¿Buscará mejorar, mantener o bajar su nivel de producción?

\section{CONCLUSIONES}

El método de regresión estableció que los datos tienen un comportamiento lineal, verificándose con su respectiva correlación que la tendencia se aproxima a Excelente.

\footnotetext{
2 Martínez Bencardino, Ciro Estadística y muestreo / Ciro Martínez Bencardino. 13 $3^{\mathrm{a}}$. ed. -- Bogotá : EcoeEdiciones,
}

Las empresas buscan rentabilidad tomando como filosofía el bienestar financiero, pero ello implica que el uso de metodologías estadísticas que propendan a estar cerca de la realidad económica del sector, los pronósticos permiten visualizar con mucha probabilidad los objetivos a alcanzar, y se debe tener en cuenta que estos cálculos van de la mano con el uso de software dado que sin ello no puede ser posible el análisis de manera acertada.

Los pronósticos encontrados, resultado del análisis matemático dieron las siguientes estimaciones:

\begin{tabular}{|c|r|r|r|}
\hline & \multicolumn{3}{|c|}{ PRONOSTICOS } \\
\hline ANOS & VENTAS & INNENTARIOS & CUENTAS POR COBRAR \\
\hline 2014 & 457 & 96 & 78 \\
\hline 2015 & 486 & 100 & 82 \\
\hline 2016 & 514 & 105 & 86 \\
\hline 2017 & 543 & 110 & 91 \\
\hline
\end{tabular}

Datos que sugieren un aumento promedio proporcional en ventas, inventarios y cuentas por cobrar, donde deben establecerse estrategias para mejorar las ventas, el manejo de inventarios y políticas para disminuir las cuentas por cobrar dado que estas últimas permiten definir en gran parte nuevos planes de negocio.

Otra de las formas de presentar los resultados y realizar el análisis respectivo es mediante el uso de las herramientas SPSS y Excel calculado por el método de mínimos cuadrados. Información que se logró presentar de manera gráfica.

\section{REFERENCIAS}

González García, Luis. Viga Juárez, Cesar y Fierro Martinez, Santiago (2017). Artículo de investigación Prospección del riesgo operativo de las Mipymes en Colombia. Fundacion Universitaria Konrad Lorenz. Publicado por Elsevier Espana, S.L.U. https://doi.org/10.1016/j.sumneg.2017.11.004

Mejía Córdova, Guillermo (2016). Pérdida de productividad en el lugar de trabajo relacionada con el estrés financiero, Universidad Autónoma de Guadalajara-Campus Tabasco, Jalisco. http://creativecommons.org/licenses/by-nc$\mathrm{nd} / 4.0 /$

Monelos, P. Sanchez, Carlos y Lopez, Manuel (2016), Predicción del fracaso empresarial. Una contribución a la síntesis de una teoría mediante el análisis comparativo de distintas técnicas de predicción. 
Serrano Salazar, Jaime y Sánchez Delgado, Maritza (2014). Costos como factor clave en la gestión de proyectos de software, Centro de Investigación Aplicada y Desarrollo en Tecnologías de Información (CIADTI). Pamplona, Norte de Santander, Colombia. Extraído de la Revista Colombiana de Tecnologías de Avanzada. ISSN: 1692-7257.

Peñaranda Peñaranda, Marta., Suarez Castrillon, Sir Alexci., Soto Arevalo, Fanny (2014). Software de aplicación como estrategia de marketing. Extraído de la Revista Colombiana de Tecnologías de Avanzada. ISSN: 16927257.

Johnson R. Probabilidad y Estadística para ingenieros. Octava edición. Pearson. (2012)

Churchill, N. y Mullins, J. (2001). How Fast Can Your Company Afford to Grow?, Harvard Business Review, May (2001). Tool Kit Reprint 0105K.

Lira Briceño, Paul (2012). Métodos para Estimar el Capital de Trabajo. Parte 1 al 3. Blogs Gestión, publicados en Junio 2012. Extraído el 10 de Febrero de 2015 del sitio web: http://blogs.gestion.pe/deregresoalobasico/2011 /01/metodos-para-estimar-el-capita.html

Selpa, A. y Espinosa, D. (2009). La Gestión del Capital de Trabajo como Proceso de la Gestión Financiera Operativa. Universidad de Matanzas Camilo Cienfuegos, Departamento de Profesores de Contabilidad y Finanzas, Cuba. Extraído el 10 de Febrero de 2015 del sitio web:

http://www.elcriterio.com/revista/ajoica/ contenidos_4/ambar_selpa_y_daisy_espinosa.p df

Vaquiro C, Jose Didier (2009). Pronóstico Financiero. Asesoría y Consultoría para Pymes. Pymes Futuro. Extraído el 10 de Enero de 2018 del sitio web: http://www.pymesfuturo.com/Pronostico.htm 